\title{
Controlled hydrothermal synthesis of nickel phosphite nanocrystals with hierarchical superstructures
}

Zhanjun Gu, ${ }^{[a, b]}$ Tianyou Zhai ${ }^{[a]}$ Bifen Gao, ${ }^{[a]}$ Damei Ke, ${ }^{[a]}$, Ying Ma ${ }^{*[a]}$ and Jiannian Yao ${ }^{*[a]}$

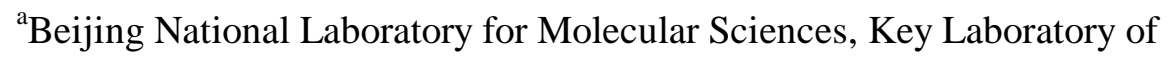

Photochemistry, Institute of Chemistry, Chinese Academy of Sciences, Beijing

(100080), P. R. China.

${ }^{\mathrm{b}}$ Graduate school of the Chinese Academy of Science, Beijing (100080), P. R. China.

E-mail: jnyao@iccas.ac.cn (J. N. Yao).

Abstract: The large-scale synthesis of the hexagonal $\mathrm{Ni}_{11}\left(\mathrm{HPO}_{3}\right)_{8}(\mathrm{OH})_{6}$ nanocrystallites with delicate morphologies, such as nanoparticles, ellipsoidal rods, dumbbell-like and urchin-like superstructures based on nanorods as well as hollow spheres made of nanoparticles have been prepared via a very simple hydrothermal method without any templates or catalyst. It is found that the morphology of the final products is strongly dependent on the reaction conditions, such as $\mathrm{pH}$, temperature and reaction times. A "rod-to-dumbbell-to-sphere" growth mechanism has proposed for the formation of the urchin-like superstructures.

Corresponding author. Fax (+86)10-82616517; Tel: (+86)10-82616517. E-mail: jnyao@iccas.ac.cn (J. N. Yao). 